\title{
VALIAÇÃo dA DIVERSIDADE GENÉTICA DE SITOPHILUS ZEAMAIS MOTSCHULSKY, 1855 (COLEOPTERA: CURCULIONIDAE) UTILIZANDO ISOENZIMAS EM AMOSTRAS DE DIVERSAS REGIÕES do Brasil e do Paraguai
}

\section{Danielle das Neves Bespalhok}

Universidade Estadual de Maringá, Departamento de Agronomia, Programa de PósGraduação em Genética e Melhoramento, Maringá, Paraná, Brasil, dani_bespalhok@hotmail.com.

\section{Bruno Cesar Circunvis}

Universidade Estadual de Maringá, Departamento de Agronomia, Programa de PósGraduação em Genética e Melhoramento, Maringá, Paraná, Brasil, bc.circunvis@gmail.com.

\section{Alison Henrique Ferreira Julio \\ Universidade Estadual de Maringá, Departamento de Bioquímica, Programa de Pós- Graduação em Biologia Celular e Molecular, Maringá, Paraná, Brasil, alisonhfj@gmail.com.}

\section{Sandro Daniel Drosdoski}

Universidade Estadual de Maringá, Departamento de Bioquímica, Programa de PósGraduação em Biologia Celular e Molecular, Maringá, Paraná, Brasil, sandro.twister@gmail.com.

\section{ERasmo Renesto}

Universidade Estadual de Maringá, Departamento de Biotecnologia, Genética e Biologia Celular, Maringá, Paraná, Brasil, erenesto@hotmail.com.

\begin{abstract}
Resumo: $O$ uso do inseticida ainda tem sido utilizado em grande escala para o controle de diversas pragas de grãos armazenados representando grandes riscos no desenvolvimento de resistências. Existem vários estudos com o Sitophilus zeamais que buscam conter suas populações, contudo estudos que analisam sua variabilidade genética é muito pouco. O objetivo deste trabalho foi o de avaliar a variabilidade genética de $S$. zeamais em 10 amostras de diversos locais, destes, oito localidades são do território Brasileiro: Tunápolis (SC), Iguatemi (PR), Piracicaba (SP), Coimbra (MG), Maracaju (MS), Canarana (MT), Jataí (GO), Picos (PI) e duas localidades são do Paraguai: Amambay e Pedro Juan Caballero. Foram empregadas metodologias de eletroforese de isoenzimas, em gel de amido para examinar seis sistemas enzimáticos (AAT, ACP, GPI, IDH, MDH e ME) e em gel de poliacrilamida que permitiram analisar a enzima (EST). A conclusão deste, foi que a espécie revelou ter uma elevada variabilidade genética $(\mathrm{He})$, uma moderada diferenciação entre as amostras e estas foram compiladas em três grupos.
\end{abstract}

Palavras-chave: eletroforese, inseto-praga, variabilidade genética. 


\title{
Evaluation of the genetic diversity of Sitophilus Zeamais Motschulsky, 1855 (Coleoptera: Curculionidae) USing isozymes in SAMPLeS FROM SOME Regions OF Brazil and Paraguay
}

\begin{abstract}
The use of insecticide has still been used in large scale for the control of several stored grain pests representing great risks in the development of resistance. There are several studies with Sitophilus zeamais that seek to contain their populations, but studies that analyze their genetic variability are very few. The objective of this work was to evaluate the genetic variability of $S$. zeamais in 10 samples from several sites, of these, eight localities are of the Brazilian territory: Tunápolis (SC), Iguatemi (PR), Piracicaba (SP), Coimbra Maracaju (MS), Canarana (MT), Jataí (GO), Picos (PI) and two localities are from Paraguay: Amambay and Pedro Juan Caballero. We used isozyme electrophoresis methodologies in starch gel to examine six enzymatic systems (AAT, ACP, GPI, HDI, MDH and ME) and in polyacrylamide gel that allowed to analyze the enzyme (EST). The conclusion of this study was that the species showed high genetic variability ( $\mathrm{He})$, a moderate differentiation between the samples and these were compiled in three groups.
\end{abstract}

Keywords: electrophoresis, insect pests, genetic variability.

\section{INTRODUÇÃO}

(O) sexto levantamento de grãos divulgado pela Companhia Nacional de Abastecimento (CONAB) no mês de fevereiro do ano de 2016 revelou que a produção de grãos no Brasil na safra 2015/2016 foi de aproximadamente 210,3 milhões de toneladas. De acordo com o documento, o volume é $1,3 \%$ superior à safra anterior (CONAB, 2016). De acordo com esse levantamento, dos 210,3 milhões de toneladas de grãos produzidos, aproximadamente 39,73 \% são de milho. E a cultura do milho é a maior em volume de produção em escala mundial, sendo os Estados Unidos, China, Brasil e Argentina os maiores produtores, o que representa $70 \%$ da produção conforme Peixoto (2014). O milho ainda será o alimento mais importante, visto que há uma estimativa de que teremos de alimentar o mundo, que atualmente possui uma população em torno de sete bilhões de pessoas e, que em 2050 excederá a nove bilhões (Peixoto, 2014).

Uma parte relevante das safras agrícolas de grãos se perde todo ano devido às falhas na colheita, no transporte e armazenamento. A situação se agrava com a falta ou uso indevido de medidas de controle de pragas, sendo essas responsáveis pelas maiores perdas (Campos, 2014). Estima-se que no Brasil 20\% da produção anual de grãos se perde entre a colheita e o armazenamento e que metade dessas perdas seja ocasionada durante o armazenamento dos grãos devido ao ataque de pragas (Campos, 2014).

Uma das principais pragas dos grãos armazenados em regiões tropicais é o Sitophilus zeamais Motschulsky, 1855 e é considerada a praga mais importante do milho no Brasil
(Guedes, 1991). Para Mckenzie (1996) o manejo da maioria das pragas de grãos armazenados tem sido feito, preferencialmente, com o uso do inseticida fumigante fosfeto de alumínio, o que representa um enorme risco no desenvolvimento de resistência a este produto. $O$ uso frequente de inseticida somado às técnicas de aplicação inapropriadas favorece 0 aumento da pressão de seleção de indivíduos resistentes (Mckenzie, 1996). Estes produtos químicos de ação prolongada podem permanecer nas águas e no solo por décadas, infiltrando-se até os lençóis freáticos. Por serem à base de metais pesados (chumbo, cádmio, mercúrio), são capazes de persistir no ambiente e provocam alta toxicidade (Lara \& Batista, 1992).

Os problemas relacionados à utilização desses inseticidas no manejo de insetos-praga de grãos armazenados estimulam a o desenvolvimento de novas alternativas de controle, principalmente as técnicas que possam ser utilizadas em pequenas propriedades rurais, onde, segundo Santos (1993), as principais pragas são S. zeamais e roedores.

Existem muitos estudos com o S. zeamais visando seu controle, porém poucos analisando a variabilidade genética desta espécie. Alguns estudos foram feitos por Beiras \& Petitpierre (1981), Pintureau et al. (1991) e Grenier et al. (1994) que analisaram esterases de três espécies de Sitophilus da Europa. Análises de microssatélites em herança de genes nulos foram realizadas, porém em outras espécies da mesma família o Curculionidae, Pissodes strobi (Liewlaksaneeyanawin et al., 2002; Kim et al., 2006).

A avaliação da variabilidade genética em populações de pragas de culturas, bem como dos elementos que conservam esta variabilidade são muito importantes na elaboração de programas de controle mais eficazes.

De acordo com Moyle \& Leidy (1992) a va- 
riabilidade genética é a herança evolutiva de uma espécie, o conhecimento, a avaliação e a manutenção da integridade genética de determinadas espécies é um dos aspectos fundamentais em programas de preservação, manejo e controle da mesma. Perante isso, podemos inferir que as técnicas moleculares genéticas estão se tornando cada vez mais úteis na constatação da variabilidade de espécies. Informações obtidas pela análise de isoenzimas, por exemplo, apesar de remota, ainda exibe sua importância e pode contribuir para várias áreas da biologia, pois é um representativo do conhecimento básico da variabilidade genética.

Conforme a conclusão dos estudos de Ferreira \& Grattapaglia (1998) e Alfenas (2006) a eletroforese de isoenzimas é um instrumento que pode ser utilizado não somente como artifício para estimar os níveis de variabilidade genética, mas também para identificar o fluxo gênico em populações naturais, no estudo da dispersão de espécies, na análise de filogenias e hibridização natural, e também no melhoramento genético.

A variabilidade genética de uma população pode ser quantificada por meio das frequências gênicas adquiridas com a análise de isoenzimas e parte do princípio de que as proteínas são cadeias de aminoácidos que mostram a expressão de um gene ativo no DNA (Torres et al., 2004). Nesta técnica, ocorre o fracionamento de moléculas em relação ás suas cargas elétricas, seus pesos moleculares e suas conformações, em suportes contendo poros e tampões apropriados, sob a condução de um campo elétrico contínuo (Alfenas, 2006). Nestes casos, considera-se que os locos analisados retratam uma amostra aleatória do genoma e, dessa maneira, a população seria representante dessa amostra (Solferini \& Selivon, 2001).

O objetivo deste trabalho foi o de avaliar a variabilidade genética de Sitophilus zeamais em 10 amostras de diversos locais, destes, oito localidades são do território Brasileiro: Tunápolis (SC), Iguatemi (PR), Piracicaba (SP), Coimbra (MG), Maracaju (MS), Canarana (MT), Jataí (GO), Picos (PI) e duas localidades são do Paraguai: Amambay e Pedro Juan Caballero.

\section{Material e métodos}

Foram empregadas metodologias de eletroforese de isoenzimas, em gel de amido para examinar seis sistemas enzimáticos: Aspartato Amino Transferase (AAT), Fosfatase Ácida (ACP), Glucose-6-fosfato Isomerase (GPI), Isocitrato Desidrogenase (IDH), Malato Desidrogenase (MDH) e Enzima Málica (ME) e em gel de poliacrilamida que permitiu analisar uma enzima, Esterase (EST).

\section{Coleta e manutenção das amostras}

Com exceção da amostra de Iguatemi (PR) que foi coletada de amostras de milho colhido e armazenado em um galpão, o restante das amostras de $S$. zeamais foram cedidas pela Prof. a Dra Leda Rita Dantonino Faroni, da Universidade Federal de Viçosa (UFV). Essas amostras foram mantidas vivas em uma incubadora, com temperatura de 25 ${ }^{\circ} \mathrm{C}$, umidade relativa de $70 \%$ e fotoperíodo de $12 \mathrm{~h}$ com luz e 12 h sem luz, na UFV. Foram ordenadas da seguinte forma, 8 amostras de diferentes estados brasileiros e duas amostras do Paraguai: 1- Tunápolis (SC), 2- Iguatemi (PR), 3- Piracicaba (SP), 4- Coimbra (MG), 5Maracaju (MS), 6- Canarana (MT), 7- Jataí (GO), 8- Picos (PI), 9- Amambay (PYA- Paraguai) e 10- Pedro Juan Caballero (PYP-Paraguai) (Fig. 1). Após ser enviado para a Universidade Estadual de Maringá (UEM), Maringá (PR), os insetos foram colocados em frascos de vidros fechados com tecido que permite a passagem de ar, contendo arroz como dieta alimentar, e mantidos em uma sala com fotoperíodo de $12 \mathrm{~h}$ de claro e $12 \mathrm{~h}$ de escuro (LD 12:12), temperatura de $25^{\circ} \mathrm{C} \pm 2$ ${ }^{\circ} \mathrm{C}$ e umidade relativa do ar de, aproximadamente, $70 \%$.

\section{Preparo das amostras}

Para os insetos serem macerados houve uma preparação antes, eles foram anestesiados com baixa temperatura, ou seja, mantidos em gelo por 10 minutos. Em seguida foram macerados, com bastão de vidro, individualmente em tubo plástico do tipo Eppendorf de $1,5 \mathrm{ml}$, a $0{ }^{\circ} \mathrm{C}$ em $20 \mu \mathrm{l}$ de solução de extração composta por glicerol e $\beta$-mercaptoetanol $0,1 \%(1: 9 \mathrm{~V} / \mathrm{V})$.

\section{Preparação dos géis}

Dois tipos de géis foram feitos para a análise da diversidade genética de $S$. zeamais: Gel de Amido e Gel de Poliacrilamidaa.

\section{Eletroforese de Isoenzimas em Gel de amido}

As enzimas AAT (Aspartato Amino Transferase), ACP (Fosfatase Ácida), GPI (Glucose-6-fosfato Isomerase), IDH (Isocitrato Desidrogenase), MDH (Malato Desidrogenase) e ME (Enzima Málica) foram reveladas em gel de amido. $O$ gel foi preparado utilizando amido penetrose 50 a $17 \%$ em solução tampão, o qual é específico para cada enzima. Essa solução é utilizada tanto para o preparo do gel como para a corrida eletroforética. O tampão Tris Citrato pH 7,6 (Tris 0,1 M e Ácido Cítrico 0,028 M) foi usado para as enzimas: GPI, IDH e 


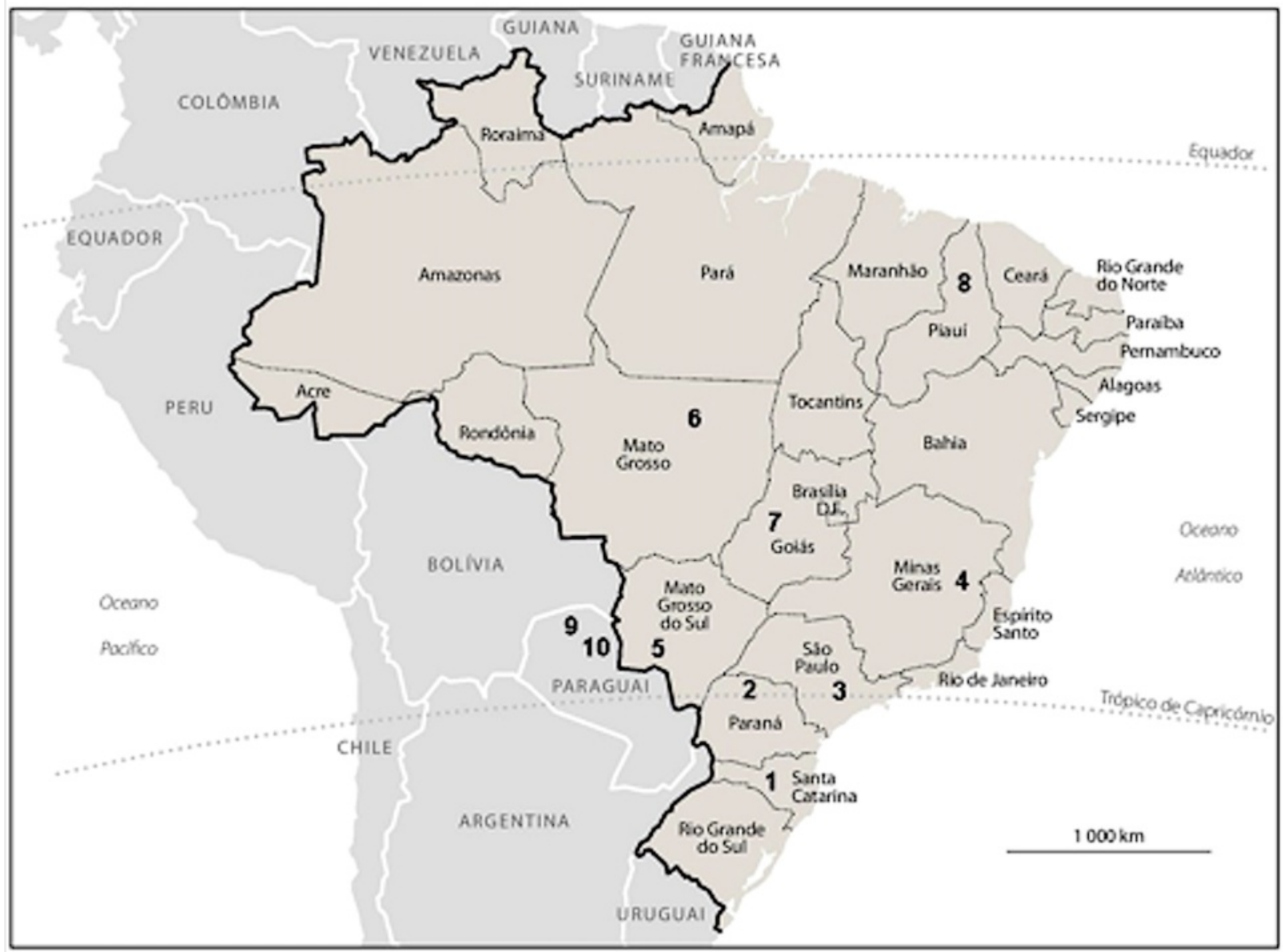

Tunápolis (SC): $26.97^{\circ} \mathrm{S}, 53.64^{\circ} \mathrm{W}$

Canarana (MT): $13.56^{\prime} \mathrm{S}, 52.27^{\prime} \mathrm{W}$

Iguatemi (PR): 23.37'S, 52.06'W

Jatai (GO): $17.88^{\circ} \mathrm{S}, 51.72^{\circ} \mathrm{W}$

Piracicaba (SP): $22.76^{\circ} \mathrm{S}, 47.84^{\circ} \mathrm{W}$

Picos (PI): $7.08^{\circ} \mathrm{S}, 41.47^{\circ} \mathrm{W}$

Fig. 1. Mapa da América do Sul com os locais de coleta de Sitophilus zeamais Motschulsky (1855). 1Tunápolis (SC), 2- Iguatemi (PR), 3- Piracicaba (SP), 4- Coimbra (MG), 5- Maracaju (MS), 6Canarana (MT), 7- Jataí (GO), 8- Picos (PI), 9- Amambay (PYA - Paraguai) e 10- Pedro Juan Caballero (PYP - Paraguai).

MDH; e o Tampão TBE pH 8,6 (Tris $0,18 \mathrm{M} /$ Ácido Bórico 0,4M e EDTA 0,004M) para as enzimas: AAT, ACP, GPI e ME.

Após o preparo das amostras, os tubos foram centrifugados em centrífuga refrigerada (Mickro 220Rd a Hettich) numa rotação de $19.0064,136 \times \mathrm{g}$, a $4{ }^{\circ} \mathrm{C}$, durante 15 minutos. Um volume de $5 \mu \mathrm{l}$ do sobrenadante foi aplicado no gel com auxílio de uma tira de papelfiltro (4 mm x $8 \mathrm{~mm}$ ) Whatman $3 M M \cap$, para cada eppendorf, o qual foi colocado numa cuba horizontal de corrida que continha o mesmo tampão usado para fazer o gel. Ligou-se a cuba a uma fonte de energia com voltagem de 250 a $300 \mathrm{~V}$ e amperagem de 30 a $40 \mathrm{~mA}$, durante um período de 17 horas, conforme Al- fenas (2006a). Após a corrida eletroforética, o gel foi fatiado horizontalmente e as fatias foram submersas em soluções histoquímicas específicas para cada sistema enzimático, preparadas segundo o protocolo de Murphy et al. (1996). Após 5 minutos, o gel foi retirado dessa solução e fixado em uma mistura de $30 \mathrm{ml}$ de álcool metílico, água destilada e ácido acético numa proporção de 5:5:1 para fixação e depois o gel foi colocado em uma placa de vidro para ser digitalizado.

\section{Eletroforese de Isoenzimas em Gel De Poliacri - LAMIDA}

A análise das Esterases foi realizada pela técnica de eletroforese descontínua em gel vertical de poliacrilamida a $11 \%$ de acordo com Davis 
(1964) e Laemmli (1970), com gel de empilhamento a $4 \%$. Em seguida ao preparo das amostras, elas foram centrifugadas por cinco minutos a $45.000 \times \mathrm{g}$ em centrífuga refrigerada a $4{ }^{\circ} \mathrm{C}$. Um volume de $15 \mu \mathrm{l}$ do sobrenadante de cada amostra foi aplicado no gel. Os géis foram submetidos à eletroforese por três horas e 30 minutos, a uma voltagem constante de $200 \mathrm{~V}$, utilizando-se para o preenchimento dos compartimentos superiores e inferiores da cuba, o tampão Tris-Glicina $0,1 \mathrm{M} \mathrm{pH} \mathrm{8,3.} \mathrm{Na} \mathrm{identificação}$ das esterases inicialmente os géis foram pré-incubados por 30 minutos em $50 \mathrm{ml}$ de tampão

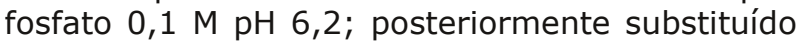
pela solução de coloração composta por tampão fosfato $0,1 \mathrm{M} \mathrm{pH} \mathrm{6,2}(50 \mathrm{ml})$, n-propanol $(5 \mathrm{ml})$, corante Fast Blue RR Salt $(0,06 \mathrm{~g})$ e os substratos a-naftil acetato $(0,03 \mathrm{~g})$ e $\beta$-naftil acetato $(0,03 \mathrm{~g})$, previamente solubilizados em acetona $(1 \mathrm{ml})$. Após uma hora de incubação no escuro à temperatura ambiente, as esterases foram visualizadas nos géis como bandas pretas ou vermeIhas, indicativas da presença de a e $\beta$-esterases, respectivamente.

\section{AnÁlise dos DAdos}

A interpretação genética foi fundamentada na estrutura quaternária das enzimas segundo Ward et al. (1992). As estimativas estatísticas foram calculadas usando o programa Popgene 1.31 (Yeh et al., 1999), tais como: a variabilidade genética avaliada de acordo com cálculo da heterozigosidade esperada $(\mathrm{He})$ e heterozigosidade observada (Ho), segundo Nei (1987). O Princípio de Hardy-Weinberg propõe a investigação dinâmica das frequências gênicas de população naturais, procurando elucidar os mecanismos que alteram a sua composição gênica ou somente a frequência genotípica pela elevação da homozigose, onde tais frequências, podem permanecerem constantes com o passar das gerações, independentemente das frequências iniciais, corroborando assim, na elucidação da matemática básica, aplicada à genética, na teoria mendeliana da hereditariedade, (Hardy, 1908; Weinberg, 1908; Crilly, 2017).

As estatísticas F (FIS, FIT e Fst) de Wright (1978), os valores das frequências alélicas, a identidade (I) e a distância genética (D) de Nei (1972).

A partir da identidade (I) e da distância genética (D) foi construído um dendrograma (método de agrupamento pelo algoritmo UPGMA - Unweighted Pair Group Method With Arithmetic Means) das amostras estudadas.

\section{Resultados e discussão}

Foi realizada análise de sete sistemas enzimáticos Aspartato Amino Transferase (AAT), Fosfatase Ácida (ACP), Glucose-6-fosfato Isomerase (GPI), Isocitrato Desidrogenase (IDH), Malato
Desidrogenase (MDH) e Enzima Málica (ME) utilizando para isso uma amostra de $S$. zeamais pertencentes à 10 localidades diferentes, o que resultou na detecção de 12 loci (Aat, Acp, Est-3, Est-4, Est-5, Est-6, Est-7, Gpi, Idh, Mdh-1, Mdh-2 e Me) e 32 alelos.

A expressão enzimática dos alelos detectados está representada nos zimogramas ilustrados pela Fig. 2. Observa-se que o alelo " $a$ " esteve presente em quase todos os loci nas 10 localidades, com exceção do loco Mdh2 que apresentou o alelo " $a$ " em apenas seis das 10 localidades. $O$ alelo " $b$ " esteve presente em todos os loci, porém apenas em alguns se fez presente nas 10 amostras, tais como Aat, Acp, Mdh-1, Mdh-2, Me e Est - 4. Já o alelo "c" apareceu nos seguintes locus Aat, Acp, Gpi, Me, Est - 3 e Est - 4, sendo que somente no locus Aat e Acp esteve presente nas 10 localidades. E por fim o alelo " $d$ " foi encontrado apenas no locus Aat e $A c p$, porém em apenas algumas localidades.

Dentre os loci visualizados também na Fig. 2 as enzimas AAT e ACP tiveram o maior número de alelos por locus (quatro) enquanto que metade das enzimas tiveram apenas dois alelos por locus nas 10 localidades analisadas.

Observaram-se também alguns alelos exclusivos, na amostra de SC, PI e PYA o alelo Aat $(d)$ com frequência de $0,053,0,040$ e 0,080 respectivamente e na amostra do PR, MT, PI, GO, PYA, PYP o alelo $A c p(d)$ com frequência de $0,021,0,065,0,020,0,019$, 0,022 e 0,059 respectivamente. Nesta situação, tais alelos poderiam ser indicados como um marcador genético para tais amostras. Dos nove alelos exclusivos, o alelo $A c p(d)$ da amostra de GO com frequência de 0,019 pode ser considerado ainda como um alelo raro, devido a sua baixa frequência.

Na Tab. 1 foram mostradas as frequências alélicas em cada locus para cada amostra analisada. Nota-se que vários loci apresentaram variação alélica e apenas os seguintes loci não apresentaram variação: Gpi das amostras de GO, PYA e PYP; Idh da amostra de MT; Mdh-2 das amostras de SP, PI, GO e PYP; Est3 da amostra de PYP; Est-5 das amostras de MG, MS, MT, PI, GO, PYA e PYP; Est-6 das amostras de SC, MG, MS, MT, GO, PYA e PYP e Est 7 das amostras de PR, MG, MT e GO.

As estimativas das frequências alélicas foram submetidas ao teste do Qui-quadrado para verificar se a população estaria em Equilíbrio de Hardy-Weinberg (EHW). O único locus detectado em EHW em todas as amostras é o $M d h-2$, enquanto que os loci Aat e Me não estão em EHW nas 10 amostras.

Nenhuma amostra tem todos os loci em EHW, porém todas as amostras apresentam pelo menos dois loci em EHW, variando o locus em cada amostra. 

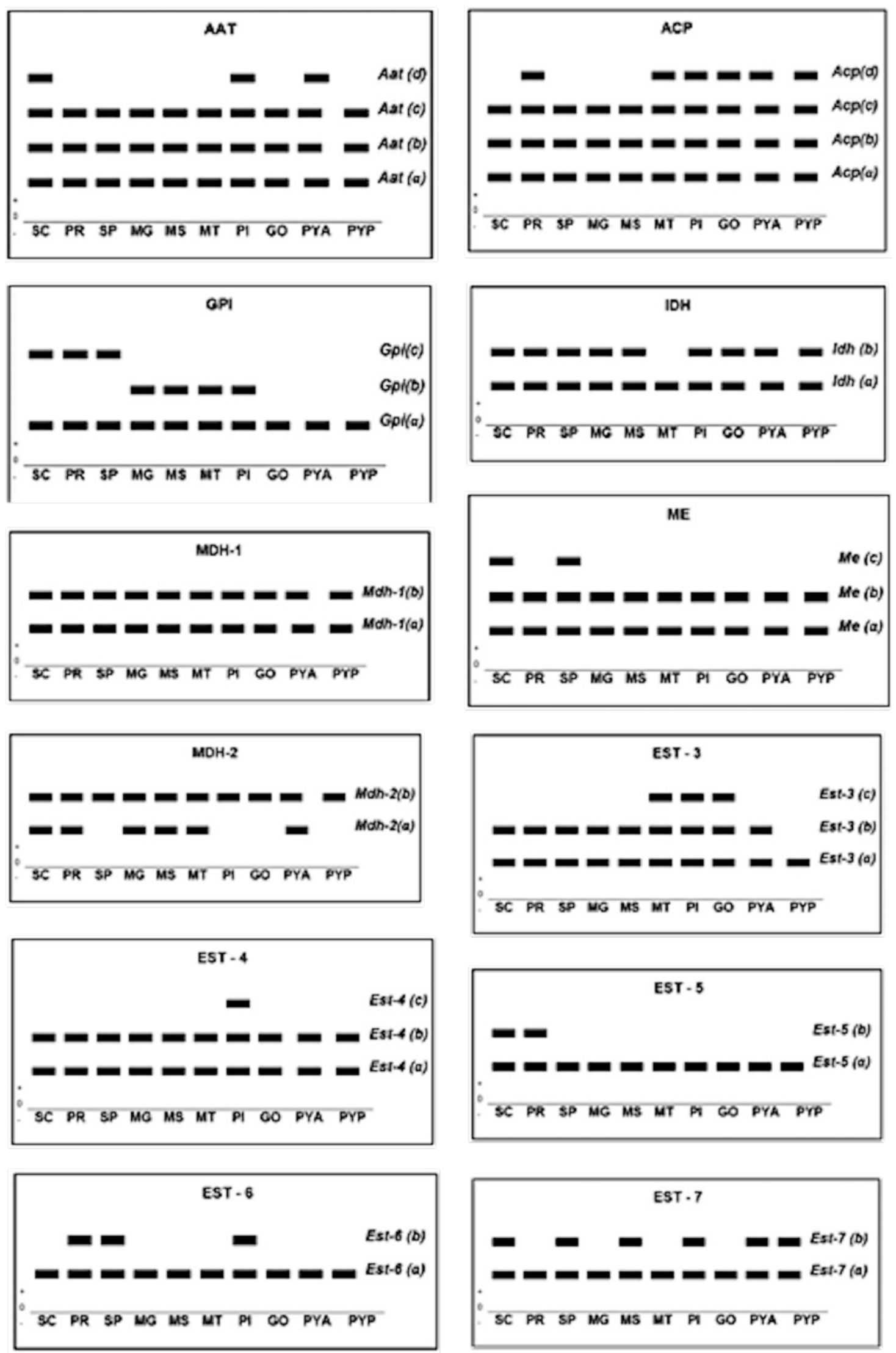

Fig. 2. Zimograma simbolizando o padrão de bandas dos sete sistemas isoenzimáticos analisados em gel de amido e poliacrilamida, com os seus 12 loci e 32 alelos, em 10 amostras de Sitophilus zeamais Motschulsky (1855). 
Tab. 1. Frequência dos alelos nas 10 amostras de Sitophilus zeamais Motschulsky (1855).

\begin{tabular}{|c|c|c|c|c|c|c|c|c|c|c|c|}
\hline Loco & Alelo & $\begin{array}{c}\text { Amostra } \\
1-\mathrm{SC}\end{array}$ & $\begin{array}{l}\text { Amostra } \\
2-P R\end{array}$ & $\begin{array}{c}\text { Amostra } \\
3-S P\end{array}$ & $\begin{array}{c}\text { Amostra } \\
4-M G\end{array}$ & $\begin{array}{c}\text { Amostra } \\
5-\mathrm{MS}\end{array}$ & $\begin{array}{c}\text { Amostra } \\
6-\mathrm{MT}\end{array}$ & $\begin{array}{c}\text { Amostra } \\
7-\mathrm{PI}\end{array}$ & $\begin{array}{c}\text { Amostra } \\
8-\mathrm{GO}\end{array}$ & $\begin{array}{c}\text { Amostra } \\
9 \text { - PYA }\end{array}$ & $\begin{array}{l}\text { Amostra } \\
10 \text { - PYP }\end{array}$ \\
\hline \multirow{4}{*}{ Aat } & $a$ & 0,237 & 0,380 & 0,480 & 0,315 & 0,568 & 0,386 & 0,440 & 0,180 & 0,280 & 0,300 \\
\hline & $b$ & 0,237 & 0,320 & 0,400 & 0,463 & 0,318 & 0,364 & 0,200 & 0,380 & 0,400 & 0,475 \\
\hline & $c$ & 0,474 & 0,300 & 0,120 & 0,222 & 0,114 & 0,250 & 0,320 & 0,440 & 0,240 & 0,225 \\
\hline & $d$ & 0,053 & $\ldots$ & $\ldots$ & $\ldots$ & $\ldots$ & $\ldots$ & 0,040 & $\ldots$ & 0,080 & $\ldots$ \\
\hline \multirow{4}{*}{$A c p$} & $a$ & 0,283 & 0,125 & 0,214 & 0,071 & 0,167 & 0,174 & 0,160 & 0,289 & 0,326 & 0,177 \\
\hline & $b$ & 0,413 & 0,375 & 0,476 & 0,429 & 0,452 & 0,304 & 0,500 & 0,289 & 0,239 & 0,353 \\
\hline & $c$ & 0,304 & 0,479 & 0,310 & 0,500 & 0,381 & 0,457 & 0,320 & 0,404 & 0,413 & 0,412 \\
\hline & $d$ & $\ldots$ & 0,021 & $\ldots$ & $\ldots$ & $\ldots$ & 0,065 & 0,020 & 0,019 & 0,022 & 0,059 \\
\hline \multirow{3}{*}{ Gpi } & $a$ & 0,963 & 0,963 & 0,963 & 0,962 & 0,962 & 0,960 & 0,962 & 1,000 & 1,000 & 1,000 \\
\hline & $b$ & $\ldots$ & $\ldots$ & $\ldots$ & 0,039 & 0,039 & 0,040 & 0,039 & $\ldots$ & $\ldots$ & $\ldots$ \\
\hline & $c$ & 0,037 & 0,037 & 0,037 & $\ldots$ & $\ldots$ & $\ldots$ & $\ldots$ & $\ldots$ & $\ldots$ & $\ldots$ \\
\hline \multirow{2}{*}{$I d h$} & $a$ & 0,974 & 0,974 & 0,972 & 0,972 & 0,921 & 1,000 & 0,952 & 0,895 & 0,905 & 0,944 \\
\hline & $b$ & 0,026 & 0,026 & 0,028 & 0,028 & 0,079 & $\ldots$ & 0,048 & 0,105 & 0,095 & 0,056 \\
\hline \multirow{2}{*}{ Mdh-1 } & $a$ & 0,929 & 0,667 & 0,563 & 0,500 & 0,667 & 0,500 & 0,611 & 0,632 & 0,650 & 0,846 \\
\hline & $b$ & 0,971 & 0,333 & 0,438 & 0,500 & 0,333 & 0,500 & 0,389 & 0,368 & 0,350 & 0,154 \\
\hline \multirow{2}{*}{ Mdh-2 } & $a$ & 0,023 & 0,019 & $\ldots$ & 0,077 & 0,077 & 0,065 & $\ldots$ & $\ldots$ & 0,135 & $\ldots$ \\
\hline & $b$ & 0,977 & 0,982 & 1,000 & 0,923 & 0,923 & 0,935 & 1,000 & 1,000 & 0,865 & 1,000 \\
\hline \multirow{3}{*}{$\mathrm{Me}$} & $a$ & 0,813 & 0,824 & 0,765 & 0,735 & 0,763 & 0,833 & 0,790 & 0,750 & 0,882 & 0,833 \\
\hline & $b$ & 0,125 & 0,177 & 0,177 & 0,265 & 0,237 & 0,167 & 0,211 & 0,250 & 0,118 & 0,167 \\
\hline & $c$ & 0,063 & $\ldots$ & 0,059 & $\ldots$ & $\ldots$ & $\ldots$ & $\ldots$ & $\ldots$ & $\ldots$ & $\ldots$ \\
\hline \multirow{3}{*}{ Est-3 } & $a$ & 0,667 & 0,433 & 0,571 & 0,316 & 0,833 & 0,816 & 0,816 & 0,870 & 0,917 & 1,000 \\
\hline & $b$ & 0,333 & 0,567 & 0,429 & 0,684 & 0,167 & 0,158 & 0,158 & 0,087 & 0,083 & $\ldots$ \\
\hline & $c$ & $\ldots$ & $\ldots$ & $\ldots$ & $\ldots$ & $\ldots$ & 0,026 & 0,026 & 0,044 & $\ldots$ & $\ldots$ \\
\hline \multirow{3}{*}{ Est-4 } & $a$ & 0,571 & 0,625 & 0,281 & 0,088 & 0,125 & 0,273 & 0,227 & 0,614 & 0,636 & 0,684 \\
\hline & $b$ & 0,429 & 0,375 & 0,719 & 0,912 & 0,875 & 0,727 & 0,568 & 0,386 & 0,364 & 0,316 \\
\hline & $c$ & $\ldots$ & $\ldots$ & $\ldots$ & $\ldots$ & $\ldots$ & $\ldots$ & 0,205 & $\ldots$ & $\ldots$ & $\ldots$ \\
\hline \multirow{2}{*}{ Est-5 } & $a$ & 0,706 & 0,947 & 1,000 & 1,000 & 1,000 & 1,000 & 1,000 & 1,000 & 1,000 & 1,000 \\
\hline & $b$ & 0,294 & 0,053 & $\ldots$ & $\ldots$ & $\ldots$ & $\ldots$ & $\ldots$ & $\ldots$ & $\ldots$ & $\ldots$ \\
\hline \multirow{2}{*}{ Est-6 } & $a$ & 1,000 & 0,778 & 0,300 & 1,000 & 1,000 & 1,000 & 0,882 & 1,000 & 1,000 & 1,000 \\
\hline & $b$ & $\ldots$ & 0,222 & 0,700 & $\ldots$ & $\ldots$ & $\ldots$ & 0,118 & $\ldots$ & $\ldots$ & $\ldots$ \\
\hline \multirow{2}{*}{ Est-7 } & $a$ & 0,600 & 1,000 & 0,960 & 1,000 & 0,587 & 1,000 & 0,500 & 1,000 & 0,200 & 0,891 \\
\hline & $b$ & 0,400 & $\ldots$ & 0,040 & $\ldots$ & 0,413 & $\ldots$ & 0,500 & $\ldots$ & 0,800 & 0,109 \\
\hline
\end{tabular}

Os alelos $a$ e $b$ foram encontrados em todas as amostras, porém com diferentes frequências (Tab. 1). Nos loci Aat, Acp, Gpi, Me, Est-3 e Est-4 foram observadas a presença do alelo c e apenas nos loci Aat e Acp foram encontrados o alelo $d$ e somente em algumaa amostras. Esse alelo $d$ pode ser considerado um alelo exclusivo, pois pode estar sendo fixado nestas amostras. De acordo com Thorpe \& Solé-Cava (1994), novos alelos aparecerão inicialmente em frequências baixas na população e o destino evolutivo dos novos alelos dependerá de 
seu desempenho fisiológico relativo (valor adaptativo ou valor seletivo) e de mudanças casuais da frequência, quando transmitido para gerações seguintes. Portanto, a provável razão da presença desses alelos exclusivos pode ser o isolamento das populações ou, ainda, devido a mutações em regiões do DNA que codificam uma determinada enzima. Assim, nas condições eletroforéticas aqui efetuadas foi possível diferenciar esse alelo, como sendo um novo alelo de frequência muito baixa, provavelmente devido à mutação. O aumento na frequência pode ser devido à seleção natural ou deriva genética (Thorpe \& Solé-Cava, 1994). Alelos exclusivos podem servir como marcadores moleculares da espécie, o que ajudará na sua identificação.

O alelo d do locus Acp na amostra de GO (Tab. 1) é o alelo que apresentou a menor frequência alélica $(0,019)$ e os de maiores frequências apareceram em vários loci de várias amostras.

Ainda utilizando as estimativas das frequências alélicas foi realizado um teste do qui quadrado de homogeneidade, que demostrou a diferenciação entre as amostras ficando evidente que dos 12 loci analisados em todas as amostras os loci Aat, Mdh-1, Mdh-2, Est-3, Est-4, Est-5, Est-6 e Est-7 diferem estatisticamente nas frequências alélicas.

A Tab. 2 mostra que a porcentagem de loci polimórficos foi maior nas amostras de SC e PR com valor de 91,67 e menor nas amostras de PI e PJC com valor de 58,33 sendo estas maiores das encontradas por Bespalhok et al. (2015), onde encontraram um valor de $30 \%$ de loci polimórficos para amostras de SC e PR, $S$. zeamais e Sitophilus oryzae respectivamente. Em estudos obtidos por Beiras \& Petitpierre (1981) a proporção de loci polimórficos foi $16 \%$ para $S$. zeamais e de acordo com Grenier et al. (1994), a porcentagem de loci polimórficos foi $89 \%$ em $S$. zeamais. A diferença de porcentagem de loci polimórficos pode ter sido o fato de que Beiras \& Petitpierre (1981) analisaram seis loci de várias enzimas enquanto que Grenier et al. (1994) analisaram apenas três loci de Esterase.

Em relação ao número médio de alelos por locus (K) (Tab. 2) verificou-se uma variação de 2,3 a 1,8 sendo que o maior número de alelos foi encontrado na amostra de GO e o menor na amostra do PJC. Bespalhok et al. (2015) obtiveram um valor médio de alelos por locus de 1,3 para amostras de $S$. zeamais. O número médio de alelos por locus aumentou em comparação com Bespalhok et al. (2015), o que ser um indicativo no aumento da variabilidade genética conforme Alfenas (2006). Nos estudos realizados por Grenier et al. (1994), foi verificado que o número médio de alelos por locus foi 2,48 nessa mesma espécie.

Conforme a Tab. 2 verificou-se a heterozigosidade esperada $(\mathrm{He})$ e a heterozigosidade observada (Ho) e constatou-se que a amostra de

Tab. 2. Medidas de variabilidade genética: K: número de alelos por loco; Ho: heterozigosidade observada; He: heterozigosidade esperada e FIS: Índice de fixação, para as 10 amostras de Sitophilus zeamais Motschulsky (1855). 1- Tunápolis (SC), 2- Iguatemi (PR), 3- Piracicaba (SP), 4- Coimbra (MG), 5- Maracaju (MS), 6- Canarana (MT), 7- Jataí (GO), 8- Picos (PI), 9- Amambay (PYA - Paraguai) e 10- Pedro Juan Caballero (PYP - Paraguai).

\begin{tabular}{ccccccc}
\hline Localidades & $\mathrm{K}$ & $\mathrm{HO}$ & $\mathrm{HE}$ & $\mathrm{F}$ IS & Loci Polimórficos & $\%$ Locus Polimórficos \\
\hline 1 - SC & 2,250 & 0,069 & 0,328 & 0,789 & 11 & $91,67 \%$ \\
2 - PR & 2,167 & 0,090 & 0,311 & 0,710 & 11 & $91,67 \%$ \\
3 - SP & 2,083 & 0,067 & 0,310 & 0,784 & 10 & $83,33 \%$ \\
$4-$ MG & 1,917 & 0,063 & 0,252 & 0,752 & 9 & $75,00 \%$ \\
5 - MS & 2,000 & 0,090 & 0,285 & 0,684 & 10 & $83,33 \%$ \\
6 - MT & 2,000 & 0,095 & 0,257 & 0,629 & 8 & $66,67 \%$ \\
7 - PI & 2,333 & 0,089 & 0,330 & 0,730 & 10 & $83,33 \%$ \\
$8-$ GO & 1,917 & 0,084 & 0,259 & 0,675 & 7 & $58,33 \%$ \\
9 -PYA & 2,083 & 0,115 & 0,291 & 0,605 & 9 & $75,00 \%$ \\
10 -PYP & 1,833 & 0,080 & 0,221 & 0,637 & 7 & $58,33 \%$ \\
\hline Média & 2,058 & 0,084 & 0,284 & 0,699 & 9,2 & $76,67 \%$ \\
\hline
\end{tabular}


Tab. 3. Comparação dos valores das médias das Heterozigosidades Esperadas (He) por locus das 10 localidades de Sitophilus zeamais Motschulsky (1855) e valores das médias de He por locus de 170 espécies de insetos do estudo de Ward et al. (1992).

\begin{tabular}{ccc}
\hline LOCO & He & Ward et al. (1972) \\
\hline AAT & 0,674 & 0,134 \\
ACP & 0,657 & 0,199 \\
GPI & 0,053 & 0,229 \\
IDH & 0,096 & 0,124 \\
MDH1 & 0,459 & $0,063^{*}$ \\
MDH2 & 0,077 & - \\
ME & 0,327 & 0,095 \\
EST3 & 0,4 & $0,274^{* *}$ \\
EST4 & 0,508 & - \\
EST5 & 0,079 & - \\
EST6 & 0,226 & - \\
EST7 & 0,326 & - \\
\hline Média & 0,322 & 0,137 \\
\hline
\end{tabular}

* Valor referente à média da enzima MDH. ** Valor referente à média da enzima EST.

GO apresentou maior He $(0,330)$ enquanto que a amostra de AM apresentou maior Ho $(0,115)$.

$\mathrm{Na}$ Tab. 3 verifica-se uma variabilidade genética elevada (Média $\mathrm{He}=0,322$ ) quando comparada à média de $\mathrm{He}$ em insetos que é de 0,137 de acordo com estudos realizados por Ward et al. (1992). Bespalhok et al. (2015) obtiveram as seguintes heterozigosidades médias esperadas:
$0,042,0,045$ e 0,00 para amostras de S. oryzae do Paraná (PR), S. zeamais de Santa Catarina (SC) e S. oryzae do Rio Grande do Sul (RS), respectivamente. No presente trabalho dos 12 loci analisados, apenas quatro loci tiveram a He menor do que a encontrada por Ward et al. (1992). A maior He encontrada foi no locus Aat com um valor de 0,674 e a me-

Tab. 4. Resumo da estatística $F$ de Wright (1978) para amostras de 10 localidades de Sitophilus zeamais Motschulsky (1855). N: tamanho da amostra; FIs: deficiência de heterozigotos em cada subpopulação; Fit: deficiência de heterozigotos nas populações totais e Fst: proporção da variabilidade genética entre populações.

\begin{tabular}{ccccc}
\hline Locus & $\mathrm{N}$ & $\mathrm{F}_{\mathrm{IS}}$ & $\mathrm{F}_{\mathrm{IT}}$ & $\mathrm{F}_{\mathrm{ST}}$ \\
\hline AAT & 235 & 0,720 & 0,734 & 0,049 \\
ACP & 224 & 0,454 & 0,469 & 0,026 \\
GPI & 259 & 1,000 & 1,000 & 1,000 \\
IDH & 189 & 0,705 & 0,712 & 0,023 \\
MDH1 & 172 & 0,924 & 0,930 & 0,076 \\
MDH2 & 249 & 0,009 & 0,060 & 0,051 \\
ME & 176 & 0,881 & 0,882 & 0,015 \\
EST3 & 166 & 0,642 & 0,722 & 0,225 \\
EST4 & 149 & 0,596 & 0,674 & 0,194 \\
EST5 & 147 & 1,000 & 1,000 & 0,231 \\
EST6 & 139 & 1,000 & 1,000 & 0,478 \\
EST7 & 149 & 0,818 & 0,893 & 0,413 \\
\hline Média & 188 & 0,697 & 0,739 & 0,139 \\
\hline
\end{tabular}


nor do locus Gpi com valor de 0,053. No trabalho realizado por Bespalhok et al. (2015) a maior He também foi encontrada para o locus Aat, com valor de 0,210 e 0,339 para amostras de $S$. oryzae (PR) e S. zeamais (SC), respectivamente.

$\mathrm{Na}$ Tab. 4 foram apresentados os valores de estatísticas $\mathrm{F}$ de Wright (1978). Médias positivas para os valores de FIs e FIT denotaram excesso de indivíduos homozigotos nas subpopulações e no total da população, respectivamente, e os valores de FST mensuram o grau de diferenciação entre as populações. Valores até 0,05 refletem uma baixa diferenciação das populações; valores entre 0,05 e 0,15 sugerem uma diferenciação moderada; entre 0,15 e 0,25 mostraram uma diferenciação alta e os valores maiores de 0,25 denotaram uma diferenciação muito alta.

Observou-se que o valor médio positivo do índice de fixação FIs $(0,697)$ e o valor de FIT $(0,739)$ para as amostras de $S$. zeamais evidencia excesso de indivíduos homozigotos, o que pode ser devido ao fato dessas amostras serem mantidas em frascos de cultura por muito tempo, o que tornou possível o acasalamento entre os indivíduos aparentados, levando a endogamia, e consequentemente, o aumento de homozigotos. No trabalho realizado por Bespalhok et al. (2015) foi encontrado um valor médio positivo do índice de fixação FIS de 0,791 e o valor de FIT 0,961 também confirmando o excesso de indivíduos homozigotos como no presente trabalho.
O valor médio para FST foi de 0,139 indicando que as amostras analisadas têm uma diferenciação genética moderada. No estudo realizado por Bespalhok et al. (2015) o valor médio para Fst foi de 0,815 indicando que as amostras de Sitophilus analisadas eram diferentes geneticamente.

A identidade genética (I) é uma das medidas usadas para verificar se duas populações são ou não da mesma espécie. Nei $(1972 ; 1987)$ produziu estatísticas para calcular a identidade e distância genética entre duas populações. Thorpe \& Solé-Cava (1994) utilizaram valores de identidade genética de Nei (1972) para a análise de populações alopátricas, abrangendo populações da mesma espécie, do mesmo gênero, de espécies diferentes e espécies de gêneros diferentes. De acordo com estes autores, o índice de identidade genética de Nei (1972) entre duas populações da mesma espécie varia entre 0,85 e 1,00 e entre duas espécies do mesmo gênero varia entre 0,35 e 0,85 . Assim, se a identidade genética entre duas unidades taxonômicas é inferior a 0,85 são duas espécies distintas. E se for inferior a 0,35 devem ser consi- deradas como espécies pertencentes a gêneros diferentes.

$\mathrm{Na}$ Tab. 5 foram apresentados valores da identidade genética (I) muito baixas e que, de acordo com Nei $(1972 ; 1987)$, evidencia que as amostras aqui estudadas são de espécies altamente parecidas, podendo-se certificar de que se trata da mesma espécie. De acordo com dados obtidos por Bespalhok et al., (2015) os valores de "I" mostraram entre as amostras do PR e SC um

Tab. 5. Identidade Genética (acima da diagonal) e distância Genética (abaixo da diagonal) (Nei, 1972). 1- Tunápolis (SC), 2- Iguatemi (PR), 3- Piracicaba (SP), 4- Coimbra (MG), 5- Maracaju (MS), 6- Canarana (MT), 7- Jataí (GO), 8- Picos (PI), 9- Amambay (PYA - Paraguai) e 10- Pedro Juan Caballero (PYP - Paraguai).

\begin{tabular}{ccccccccccc}
\hline Amostra & 1 -SC & 2-PR & 3-SP & 4-MG & 5-MS & 6-MT & 7-PI & 8-GO & 9-PYA & 10-PYP \\
\hline 1 -SC & $* * * *$ & 0,945 & 0,874 & 0,896 & 0,937 & 0,928 & 0,954 & 0,950 & 0,944 & 0,959 \\
2 -PR & 0,056 & $* * * *$ & 0,949 & 0,953 & 0,920 & 0,958 & 0,931 & 0,963 & 0,884 & 0,952 \\
3 -SP & 0,135 & 0,053 & $* * * *$ & 0,925 & 0,911 & 0,930 & 0,915 & 0,903 & 0,833 & 0,893 \\
4 -MG & 0,110 & 0,048 & 0,078 & $* * * *$ & 0,940 & 0,963 & 0,919 & 0,921 & 0,840 & 0,893 \\
5 -MS & 0,065 & 0,084 & 0,093 & 0,062 & $* * * *$ & 0,969 & 0,983 & 0,935 & 0,939 & 0,941 \\
6 -MT & 0,074 & 0,043 & 0,073 & 0,038 & 0,031 & $* * * *$ & 0,958 & 0,977 & 0,904 & 0,962 \\
7 -PI & 0,048 & 0,072 & 0,089 & 0,085 & 0,017 & 0,043 & $* * * *$ & 0,945 & 0,958 & 0,948 \\
8 -GO & 0,051 & 0,038 & 0,102 & 0,083 & 0,067 & 0,024 & 0,057 & $* * * *$ & 0,920 & 0,986 \\
9 -PYA & 0,058 & 0,124 & 0,183 & 0,174 & 0,063 & 0,101 & 0,043 & 0,084 & $* * * *$ & 0,938 \\
$10-P Y P$ & 0,042 & 0,050 & 0,113 & 0,113 & 0,061 & 0,039 & 0,054 & 0,014 & 0,065 & $* * * *$ \\
\hline
\end{tabular}




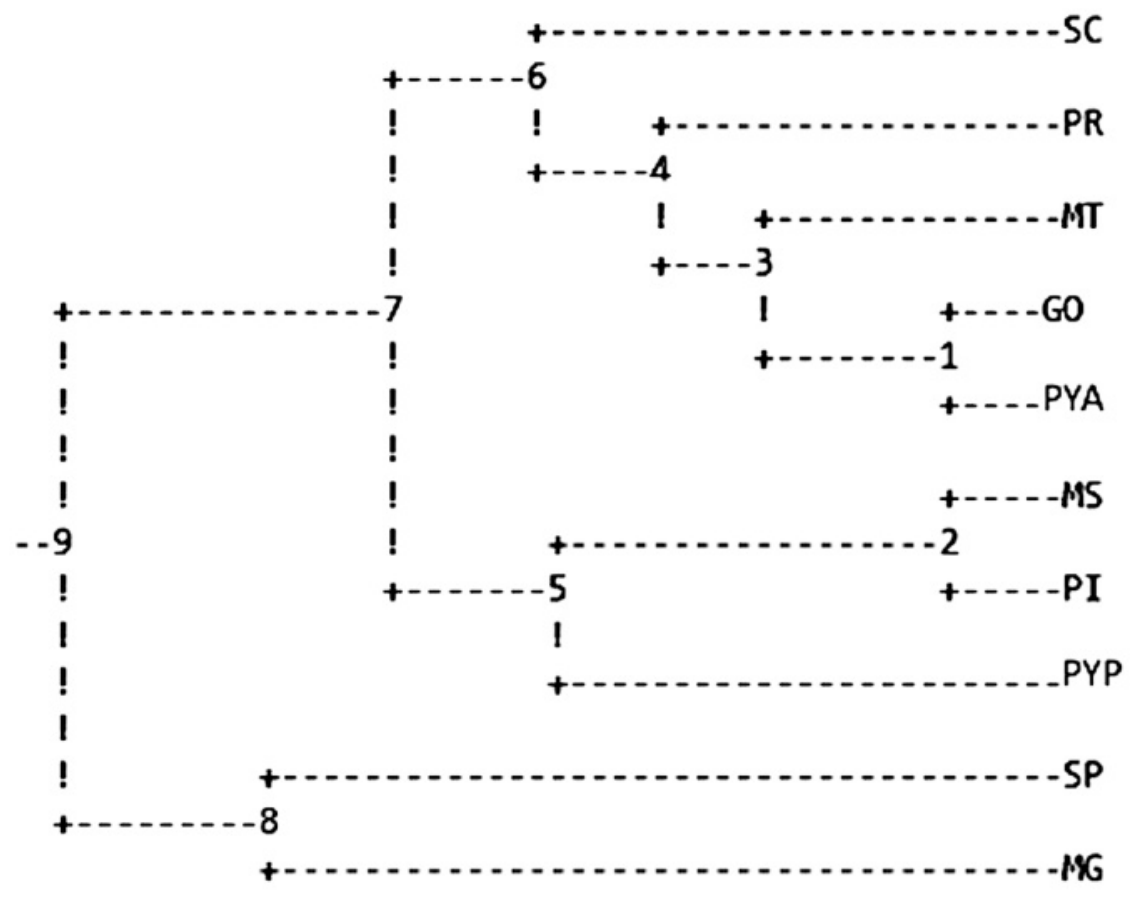

Fig. 3. Dendrograma de acordo com Nei (1972) utilizando a distância genética pelo método UPGMA. Tunápolis (SC), Iguatemi (PR), Piracicaba (SP), Coimbra (MG), Maracaju (MS), Canarana (MT), Jataí (GO), Picos (PI), Amambay (PYA - Paraguai) e Pedro Juan Caballero (PYP - Paraguai).

37 valor de 0,689 , entre PR e RS de 0,998 e entre SC e RS de 0,693 , indicando que as amostras do PR e RS são da mesma espécie enquanto que a de SC é de outra espécie.

O dendrograma apresentado na Fig. 3 mostra as relações entre as populações baseada na distância genética de Nei (1972) calculada pelo método UPGMA. Com base no dendrograma e nos testes de qui-quadrado para distâncias genéticas, pode-se verificar que as amostras pertencem a três grupos: $(S C+P R+M T+G O+P Y P)$, $(M S+P I+P Y A)$ e $(S P+M G)$.

Uma possível explicação para o excesso de homozigotos observado no presente estudo é o fato de que as amostras analisadas procedem de linhagens que provavelmente foram derivadas de um número muito pequeno de indivíduos, ou da mesma progênie. Podendo inferir, de acordo com Alfenas (2006) que a seleção natural e a deriva genética podem ter desempenhado um papel essencial na fixação aleatória de alelos e a constância ambiental do $S$. zeamais em armazéns de grãos ou em laboratório.

O estudo das estruturas populacionais por meio de técnicas moleculares é uma das partes mais importante da genética da conservação e tem sido útil tanto no estudo de populações exploradas comercialmente (abundantes, mas com riscos populacionais devido à super-exploração) como nas espécies já ameaçadas de extinção (Solé-Cava, 2001).
A utilização de marcadores moleculares foi relevante na análise das características das amostras de $S$. zeamais nas diferentes regiões do Brasil e Paraguai. Esse estudo permitiu verificar a variabilidade genética desses insetos, gerando dados importantes sobre as relações genéticas entre as populações resultando em informações favoráveis para estudos futuros que envolvam manipulação genética e manejo dessas pragas.

\section{Conclusões}

Conclui-se que dos 12 loci analisados oito tiveram valores altos de $\mathrm{He}$ constatando uma variabilidade genética elevada.

A estatística $\mathrm{F}$ de Wright mostra excesso de indivíduos homozigotos, provavelmente devido à endogamia e o FST indica que as 10 amostras são muito semelhantes geneticamente.

A identidade genética sugere que as amostras são de espécies altamente parecidas e a distância genética indica que as amostras são de espécies muito próximas geneticamente, podendo afirmar que se trata provavelmente de amostras de mesma espécie.

As amostras podem ser agrupadas em três grupos: $(S C+P R+M T+G O+P Y P)$, $(M S+P I+P Y A)$ e $(S P+M G)$. 


\section{Agradecimentos}

À Universidade Estadual de Maringá (UEM), ao Programa de Pós-Graduação em Genética e Melhoramento (PGM) e à Coordenadoria de Aperfeiçoamento de Pessoal de Ensino Superior (Capes).

\section{RefERÊNCIAS}

Alfenas, A. C. 2006a. Eletroforese e marcadores bioquímicos em plantas e microrganismos. Viçosa: UFV, $627 \mathrm{p}$.

Alfenas, A. C. 2006b. Eletroforese de isoenzimas e proteínas afins-fundamentos e aplicações em plantas e microorganismos. Viçosa: UFV, 574 p.

Allcock, A. L., M. Chauvet, K. A. Crandall, D. R. Given, S. J. G. Hall, J. M. Iriondo, T. M. Lewinsohn, S. M. Lynch, A. M. Solé-Cava, E. Stackebrandt, A. R. Templeton \& P. C. Watts. 1995. Genetic diversity as a component of biodiversity. p. 57-88. In: Heywood, V. H. \& R. T. Watson. (Eds.). Global Biodiversity Assessment. Cambridge: Cambridge University Press.

Augustin, E., A. E. Loeck, G. Storch, D. D. Grützmacher, A. P. S. Afonso \& L. G. Gusmão. 1999. Identificação de formigas cortadeiras do gênero Acromyrmex (Hymenoptera: Formicidae) através de isoenzimas. Rev. Bras. de Agroc. 5: 217220.

Avise, J. C. 1975 . Systematic value of electrophoretic data. System. Zool. 23: 465481.

Avise, J. C. 1994. Molecular markers, natural history and evolution. London: Chapman \& Hall, $493 p$.

Beiras, M. J. \& E. Petitpierre. 1981. Allozymic variability and genetic differentiation in three species of Sitophilus L. (Coleoptera, Curculionidae). Egyptian J. of Gen. and Cytolog. 10: 95-104.

Bespalhok, D. N.; E. Renesto \& T. Signorini. 2015. Enzyme Polymorphism in Sitophilus oryzae (Linaeus, 1763) and Sitophilus zeamais (Motschulsky, 1855) (Coleoptera, Curculionidae) in southern Brazil. Acta Scient. Biol. Sci. 37(2): 205211.

Borém, A. \& E. T. Caixeta. 2009. Marcadores moleculares. Viçosa: Folha de Viçosa, $531 \mathrm{p}$.
Brown, A. D. H. 1978. Isozymes, plant population, genetic structure and genetic conservation. Theor. and Appl. Gen. 52: 45-147.

Campos, T. B. 2005. Pragas dos Grãos Armazenados. Anais da XII Reunião Itinerante de Fitossanidade do Instituto Biológico - Pragas agroindustriais - Ribeirão preto - SP. Disponível em: http://www.biologico.sp.gov.br/rifib/XII\%20RIFIB\%20anais.pdf. Acesso em 12 out. 2014.

CONAB. Companhia Nacional de Abastecimento. 2016. Acompanhamento de safra brasileira: grãos, sexto levantamento, fevereiro, 2016. Disponível em: http://www.conab.gov.br/OlalaCMS/uploads/arquivos/ 16_03_11_15_20_36_boletim_graos_marco_2016.pdf. Acesso em 22 mar. 2016.

Cotton, R. T. \& D. A. Wilbur. 1982. Insects. p. 281-318. In: Christensen C. M. Stored of cereal grains and their products. St. Paul. Minnesota, A. O. C. S., cap. 9.

Crilly, T. 2017. 50 Ideias de Matemática que Você Precisa Conhecer. $1^{\circ}$ ed. São Paulo: Planeta, $216 \mathrm{p}$.

Davis, B. J. 1964. Disc electrophoresis II. Methods and application to human serum proteins. Ann. of the New York Acad. of Sci. 721: 404-427.

EMBRAPA. 2012. Manejo Integrado de pragas de grãos armazenados. Disponível em: http:// www.cnpt.embrapa.br/pesquisa/entomologia /mip_sitoph.htm. Acesso em 29 de jun. 2012.

Evans, D. E. 1981. The biology of stored products Coleoptera. p. 145-185. In: Proc. Aust. Dev. Asst. Course on Preservation of Stored Cereals.

Ferreira, M. E. \& D. Grattapaglia. 1998. Introdução ao uso de marcadores moleculares em análise genética. Brasília: EMBRAPA-CENARGEN, $220 \mathrm{p}$.

Futuyma, D. J. 1992. Biologia evolutiva. Ribeirão Preto: Sociedade Brasileira de Genética, $646 \mathrm{p}$.

Gallo, D., O. Nakano, S. Silveira Neto, R. P. L. Carvalho, G. C. Batista, E. Berti Filho, J. R. P. Parra, R. A. Zucchi, S. B. Alves \& J. D. Vendramin. 2002. Entomologia Agrícola. Piracicaba: Biblioteca de Ciências Agrárias Luiz de Queiroz, FEALQ, 920 p. 
Grenier, A. M., B. Pintureau \& P. Nardon. 1994. Enzymatic variability in 3 species of Sitophilus (Coleoptera, Curculionidae). J. of Stored Prod. Res. 30: 201-213.

Guedes, R. N. C. 1990. Manejo integrado para a proteção de grãos armazenados contra insetos. Rev. Bras. Armaz. 15: 3-48.

Guedes, R. N. C. 1991. Resistência a inseticidas: desafio para o controle de pragas de grãos armazenados. Seiva. 50: 24-29.

Guedes, R. N. C., J. O. L. Lima, J. P. Santos \& C. D. Cruz. 1995. Resistance to DDT and pyrethroids in Brazilian populations of $\mathrm{Si}$ tophilus zeamais Motsch. (Coleoptera: Curculionidae). J. Stored Prod. Res. 31: 145-150.

Guedes, R. N. C. 1999. Resistência de insetos a inseticidas. p. 101-107. In: Zambolim, L. I Encontro de sobre manejo integrado de doenças e pragas. Viçosa: UFV.

Hamrick, J. L. \& M. J. W. Godt. 1990. Allozyme diversity in plant species. p. 43-63. In: Brown, H. D., M. T. Clegg, A. L. Kahler \& B. S. Weir. (Eds.). Plant population genetics, breeding and genetic resources. Sunderland: Sinauer Associates Inc.

Hardy, G. H. 1908. Mendelian proportions in a mixed population. Science. 78: 49-50.

Hickman JR. C.P., L. S. Roberts \& A. Larson. 2004. Princípios integrados de zoologia. Rio de Janeiro: Koogan, 839 p.

Hidayat, P. \& T. W. Phillips, R. H. FfrenchConstant. 1996. Molecular and morphological characters discriminate Sitophilus oryzae and $S$. zeamais (Coleoptera: Curculionidae) and confirm reproductive isolation. Ann Entomol. Soc. Am. 89(5): 645652.

Hunter, R. L. \& C. L. Markert. 1957. Histochemical demonstration of enzymes separated by zone electrophoresis in starch gels. Science. 125: 1294-1295.

Kim, K. S., P. Cano-Rios \& T. W. Sappington.. 2006. Using genetic markers and population assignment techniques to infer origin of boll weevils (Coleoptera: Curculionidae) unexpectedly captured near on eradication zone in Mexico. Envir. Entomol. 35: 813-826.

Kuschel, G. 1961. On problems of synonymy in the Sitophilus oryzae complex (30 th contribuition. Coleoptera, Curculionidae). Ann. Mag. Nat. Hist. 13: 241-244.
Laemmli, U. K. 1970. Cleavage of structural proteins during the assembly of the head of bacteriophage T4. Nature. 227(5259): 377-384.

Lara, M. A. C. 1998. Variabilidade genética em bovinos e bubalinos a través de polimorfismos protéicos: análise populacional e suas implicações no melhoramento. Ribeirão Preto: Universidade de São Paulo, Tese (Doutorado em Genética), 215 p.

Lara, M. A. C., J. R. Sereno, U. G. P. Abreu, F. T. P. S. Sereno \& E. P. B. Contel. 2001. Estudio preliminar de relaciones geneticas entre razas naturalizadas brasileñas, cebuínas y europeas. Arch. de Zootec. 50: 165-170

Lara, W. H. \& G. C. Batista. 1992. Pesticidas. Química Nova. 15: 161-166.

Liewlaksaneeyanawin, C., C. E. Ritland \& Y. A. El-Kassaby. 2002. Inheritance of null alleles for microsatellites in the white pine weevil (Pissodes scrobe [Peck] Coleoptera: Curculionidae). J. of Hered. 93: 67-70.

Lorini, I. 2008. Manejo integrado de pragas de grãos de cereais armazenados. Passo Fundo: Embrapa Trigo, 72 p.

Market, C. L. 1975 . Biology of isozymes. In: Market, C. L. Isozymes: molecular structure. New York: Academic Press, p. 1-9.

Marques, D. K. S. 2002. Aplicação da biologia molecular em programas de conservação de recursos pesqueiros. Corumbá: Embrapa Pantanal, 22 p.

McKenzie, J. A. 1996. Ecological and evolutionary aspects of insecticide resistance. Acad. Press, $185 \mathrm{p}$.

Moyle, P. B. \& R. A. Leidy. 1992. Loss of biodiversity in aquatic ecosystems: evidence from fish faunas. p. 127-169. In: Fielder, P. L. \& S. K. Jain. (Eds.). Conservation Biology: the theory and practice of nature conservation, preservation and management. New York: Chapman and Hall.

Murphy, R. W., J. W. Sites, D. G. Buth \& C. H. Haufler. 1996. Proteins: isozyme electrophoresis. p. 51-120. In: Hillis, D. M., C. Moritz \& B. K. Mable. (Eds.). Molecular systematics. Massachusetts: Sinauer Associates. 
Nei, M. 1972. Genetic distance between populations. Am. Natural. 106: 283-291.

Nei, M. 1987. Molecular evolutionary genetics. Columbia University Press, New York, p. 512.

Peixoto, C. M. 2014. O milho no Brasil, sua importância e evolução. DuPont Pioneer, 2014. Disponível em: http://www.pioneersementes.com.br/Media-Center/Pages/ Detalhe-do-Artigo.aspx $? \mathrm{p}=165 \mathrm{\& t}=\mathrm{O}+\mathrm{mi}-$ Iho+no+Brasil\%2c+sua+import\%u00e2ncia +e+evolu\%u00e7\%u00e 3o. Acesso em 12 out. 2014.

Pereira, P. R. V. S. \& J. R. Salvadori. 2006. Identificação dos principais Coleoptera (Insecta) associados a produtos armazenados. Passo Fundo: Embrapa Trigo, 33 p.

Pierce, L.C. \& J.L. Brewbaker. 1973. Applications of isozyme analysis in horticultural science. Hort. Sci. 8: 17-22.

Pintureau, B., A. M. Grenier \& P. Nardon. 1991. Polymorphism of esterases in 3 species of Sitophilus (Coleoptera, Curculionidae). J. of Stored Prod. Res, 27: 14115.

Rees, D. P. 1996. Coleoptera. p. 1-3. In: Subramanyam, B. H. \& D. W. Hagstrum. (Eds.). Integrated management of insects in stored products. New York: Marcel Dekker.

Ribeiro-Costa, C. S. \& R. M. Rocha. 2006. Invertebrados: manual de aulas práticas. Riberão Preto: Holos, 285 p.

Rosseto, C. J. 1969. O complexo de SitophiIus spp. (Coleoptera: Curculionidae) no Estado de São Paulo. Bragantia. 28(10): 127-148.

Rosseto, C. J. 1972. Resistência de milho a pragas da espiga, Helicoverpa zea (Boddie), Sitophilus zeamais Motschulsky e Sitotroga cerealella (Olivier). Tese-Doutorado, Piracicaba, ESALQ, $144 \mathrm{p}$.

Santos, J. P. 1993. Recomendações para o controle de pragas de grãos e de sementes armazenadas. p. 197-236. In: Büll, L. T. \& H. Cantarella. (Eds.). Cultura do miIho: fatores que afetam a produtividade. Piracicaba: Potafos.
Santos, J. P., I. V. M. Cajueiro \& R. A. Fontes. 1986. Controle de pragas no milho armazenado em paióis. p. 66-67. In: EMBRAPA, Relatório técnico anual do Centro Nacional de pesquisa de Milho e Sorgo 1980/1984. Sete Lagoas: CNPMS/EMBRAPA.

Santos, R. F., R. D. Lacerda, A. R. Redondo, A. M. A. Nascimento, C. E. Souza, L. E. Borba, A. R. Ribeiro \& B. M. Lovato. 2012. Diversidade Genética. Disponível em: www.icb.ufmg.br/lbem/pdf/santos09biotaminas-divgen.pdf. Acesso em 28 mar. 2012.

Scandalios, J. G. 1969. Genetic control of multiple molecular forms of enzymes in plant: a review. Bioch. Gen. 3: 37-79.

Scandalios, J. G. 1975. Genes, isozymes and evolution. p. 1-7. In: Market, C. L. Isozymes: genetics and evolution, New York: Academic Press, v. 4.

Silva A. A., L. S. Braga, R. N. C. Guedes \& M. G. Tavares. 2015. Cytogenetic analyses using C-banding and DAPI/CMA3 staining of four populations of the maize weevil Sitophilus zeamais Motschulsky, 1855 (Coleoptera, Curculionidae). Comp. Cytogen. 9(1): 89102.

Smithies, O. 1955. Zone etectrophoresis in starch gels: group variations in the serum proteins of normal human adults. Bioch. J. 6: 629-641.

Solé-Cava, A. M. 2001. Biodiversidade molecular e genética da conservação. p. 173-192. In: Matioli, S. R. (Ed.). Biologia molecular e evolução. Ribeirão Preto: Holos.

Solferini, V. N. \& D. Selivon. 2001. Polimorfismo de isozimas. p. 137-142. In: Matioli, S. R. (Ed.). Biologia Molecular e Evolução. Ribeirão Preto: Holos.

Stresser, R. 2014. Pragas: principais insetos que atacam grãos e outros produtos estocados Sitophilus zeamais. Disponível em: http:// www.tecnigran.com.br/index. php/pragas/8prejuizos-causados-pelos-insetos-em-graose-outros-produtos-armazenados. Acesso em 15 out. 2014.

Teetes, G. L., K. W. Seshu Reddy, K. Leuschener \& L. R. House. 1983. Sorghum insect identification hand book. Icrisat Inf. Bull. 12: 106-107. 
Thorpe, J. P. \& A. M. Solé-Cava. 1994. The use of allozyme electrophoresis in invertebrate systematics. Zool. Scripta. 23: 3-18.

Torres, R. A., D. A. Matoso \& R. F. Artoni. 2004. Genética de peixes neotropicais. II. Biologia molecular de peixes neotropicais. Ciências Biológicas e Saúde, 10: 27-37.

Ward, D. R., D. O. F. Skibinski \& M. Woodwark. 1992. Protein heterozygosity, protein structure, and taxonomic differentiation. Evol. Biol. 26: 73-159.

Weinberg, W. 1908. Über den Nachweis der Vererbung beim Menschen. Jahresh. Verein $\mathrm{f}$. vaterl. Naturk. Würtem. 64: 368-382.

Wright, S. 1978. Evolution and genetics of populations. Chicago: The University of Chicago Press, $465 \mathrm{p}$.

Yeh, F. C., R-C. Yang, T. B. J. Boyle, Z-H. Ye \& J. X. Mao. 1999. POPGENE, the User-friendly Shareware for Population Genetic Analysis. Molecular Biology and Biotechnology Centre, University of Alberta, Canada.

Zawadzki, C. H., E. Renesto, R. E., M. O. M. Reis \& R. P. Mateus. 2005. Allozyme relationships in hypostomines (Teleostei: Loricariidae) from the Itaipu Reservoir, upper Rio Paraná basin, Brazil. Genetic. 123: 271-283.

Recebido em 16.V.2017 Aceito em 31.V.2019 